\title{
Recent results for the proton spin decomposition from lattice QCD
}

\section{Constantia Alexandrou ${ }^{a b}$, Martha Constantinou ${ }^{a b}$, Kyriakos Hadjiyiannakou $^{c}$, Christos Kallidonis $^{b}$, Giannis Koutsou ${ }^{b}$, Karl Jansen ${ }^{d}$, Haralambos Panagopoulos ${ }^{a}$, Fernanda Steffens $^{d}$, Alejandro Vaquero ${ }^{e}$, Christian Wiese ${ }^{* d}$}

${ }^{a}$ Department of Physics, University of Cyprus, P.O. Box 20537, 1678 Nicosia, Cyprus

${ }^{b}$ Computation-based Science and Technology Research Center, Cyprus Institute, 20 Kavafi Str., Nicosia 2121, Cyprus

${ }^{c}$ Department of Physics, The George Washington University, Washington, DC 20052, USA

${ }^{d}$ John von Neumann Institute for Computing (NIC), DESY, Platanenallee 6, D-15738 Zeuthen, Germany

${ }^{e}$ INFN Sezione di Milano-Bicocca, Edificio U2, Piazza della Scienza 3, 20126 Milano, Italy

E-mail: christian.wiese@desy.de

\begin{abstract}
The exact decomposition of the proton spin has been a much debated topic, on the experimental as well as the theoretical side. In this talk we would like to report on recent non-perturbative results and ongoing efforts to explore the proton spin from lattice QCD. We present results for the relevant generalized form factors from gauge field ensembles that feature a physical value of the pion mass. These generalized form factors can be used to determine the total spin and angular momentum carried by the quarks. In addition we present first results for our ongoing effort to compute the angular momentum of the gluons in the proton.
\end{abstract}

XXIV International Workshop on Deep-Inelastic Scattering and Related Subjects

11-15 April, 2016

DESY Hamburg, Germany

\footnotetext{
* Speaker.
} 


\section{Introduction}

The understanding of the proton spin decomposition is an important topic within the nucleon structure community. In particular, this is motivated by the experimental measurement and analysis of the spin dependent structure function of the proton $g_{1}^{p}$ by the European Muon Collaboration [1], which showed that the quark spin only contributes about half the value of the proton's total spin. Usually, these quantities are extracted from spin dependent structure functions which are obtained from polarized scattering experiments as they have for instance been performed at SLAC, JLab, CERN and DESY. Unfortunately, there are not as many data points available as for unpolarized DIS and consequently the uncertainties for the extracted structure functions, especially for the gluon, are rather large (see e.g. Ref. [2]).

Thus, an alternative determination of these quantities from first principles would certainly be most useful and moreover provide a rigorous test to the theory of QCD. For this task lattice QCD is a promising candidate, since it is able to make ab initio prediction for QCD observables in various energy regimes. This includes matrix elements of local operators, which are an essential tool for studying nucleon structure. In particular these matrix elements can be related to the form factors relevant for the nucleon spin and thus have the potential to reveal the spin structure from a first principle lattice calculation.

\section{The proton spin}

One gauge invariant possibility to decompose the proton spin was proposed in Ref. [3] and is widely known as Ji's sum rule. It decomposes the proton spin in the following parts

$$
\frac{1}{2}=\frac{1}{2} \Delta \Sigma+L_{q}+J_{g}
$$

with the intrinsic quark spin $\frac{1}{2} \Delta \Sigma$, the total quark orbital angular momentum $L_{q}$ and the gluon angular momentum $J_{g}$. The sum of the first two give the total angular momentum of all quarks $J_{q}=\frac{1}{2} \Delta \Sigma+L_{q}$. Unlike $J_{q}, J_{g}$ cannot be further decomposed in a gauge invariant way. The quark angular momentum can be expressed as the sum of two proton form factors

$$
J_{q}=\sum_{q} \frac{1}{2}\left(A_{20}^{q}(0)+B_{20}^{q}(0)\right)
$$

which are defined as moments of certain generalized parton distributions (GPDs)

$$
A_{20}^{q}(0)=\int_{-1}^{1} d x x H^{q}(x, 0,0)=\langle x\rangle_{q}, \quad B_{20}^{q}(0)=\int_{-1}^{1} d x x E^{q}(x, 0,0) .
$$

where $\langle x\rangle_{q}$ is the average quark momentum fraction in the proton. A review on the various GPDs can be found in Ref. [4]. The gluon angular momentum $J_{g}$ can be expressed correspondingly. The quark spin $\frac{1}{2} \Delta \Sigma$ can be related to the proton singlet axial charge

$$
\Delta \Sigma=g_{A}^{(0)}=\sum_{q} \tilde{A}_{10}^{q}(0)=\sum_{q} \int_{-1}^{1} d x \tilde{H}^{q}(x, 0,0) .
$$

From the phenomenological analysis of experimental data there are estimates of parts of the spin decomposition for instance for the quark spin $0.13<\frac{1}{2} \Delta \Sigma<0.18$ [2] or the total quark angular momentum $0.24<J^{q}<0.30$ [5]. 


\section{Lattice setup}

On the lattice we can extract form factors from proton matrix elements of certain operators $\langle P|\mathscr{O}| P\rangle[6]$. For the spin decomposition these are the local axial-vector and one-derivative vector operators

$$
\mathscr{O}_{A}^{\mu, q}=\bar{q} \gamma^{\mu} \gamma^{5} q \quad O_{V}^{\mu v, q}=\bar{q} \gamma^{\{\mu} D^{\nu\}} q
$$

with $D^{v}$ being the covariant forward and backward derivative and $\{\ldots\}$ representing symmetrization and subtraction of trace.

There are the following relation between these matrix elements and the relevant form factors

$$
\begin{gathered}
\left\langle P\left|\mathscr{O}_{A}^{\mu, q}\right| P\right\rangle=\bar{u}(P) \tilde{A}_{10}^{q}(0) \gamma^{\mu} \gamma^{5} u(P) \\
\left\langle P\left|\mathscr{O}_{V}^{\mu v, q}\right| P^{\prime}\right\rangle=\bar{u}(P)\left(A_{20}^{q}(t) \gamma^{\{\mu} \bar{P}^{v\}}+B_{20}^{q}(t) \frac{\sigma^{\{\mu \rho} \Delta_{\rho} \bar{P}^{v\}}}{2 m}+C_{20}^{q}(t) \frac{\Delta^{\{\mu} \Delta^{v\}}}{m}\right) u(P)
\end{gathered}
$$

where $\bar{P}=\frac{P+P^{\prime}}{2}, \Delta=P-P^{\prime}$ and $t=\Delta^{2}$. In the limit $P=P^{\prime}$ only the $A_{20}^{q}$ form factor remains.

For the gluon content one can use the gluon energy momentum tensor in order to extract the relevant form factor

$$
\left\langle P\left|\mathscr{O}_{V}^{\mu v, g}\right| P\right\rangle=\bar{u}(P) A_{20}^{g}(0) \gamma^{\{\mu} P^{v\}} u(P), \quad \text { with } \quad O_{V}^{\mu v, g}=G^{\{\mu \rho} G_{\rho}{ }^{v\}}
$$

where $G^{\mu v}$ is the field strength tensor. Certainly, $B_{20}^{g}$ contributes to the gluon angular momentum as well, but cannot be computed at the moment and is thus neglected. These matrix elements of operators can be related to the ratio between three- and two-point correlation functions. The correlation function is constructed from interpolating fields of the proton and the operator [6]. Free quark fields have to be contracted to quark propagators using Wick's theorem. Depending on the choice of the operator there are different possibilities to perform the contractions. They can be categorized into three general types of contractions, as shown in Fig. 1. All depicted types of contractions have
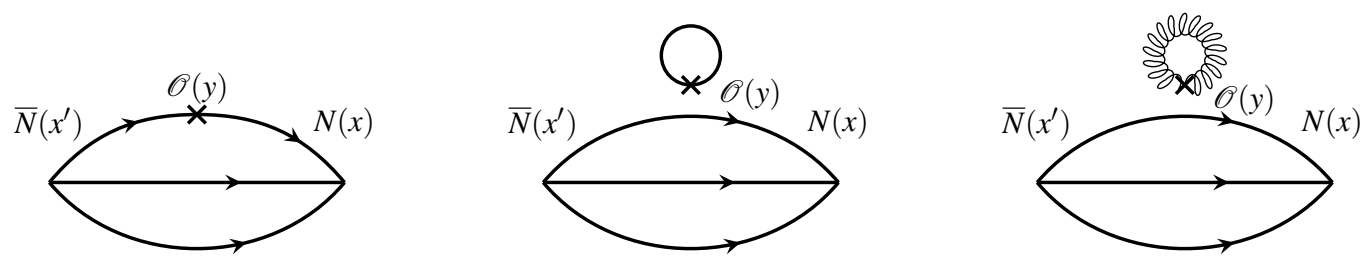

Figure 1: Possible Wick contractions of three-point correlation functions for the spin decomposition. Left: connected middle: disconnected quark loop or right: gluon loop.

to be considered in order to explore the spin composition. While the here considered light quark form factors will have connected and disconnected contractions, one will find only quark disconnected contractions for the strange and heavier quarks and the gluon disconnected contraction for the gluon contribution to the spin.

All results that will be presented in this work are computed on the latest European Twisted Mass Collaboration (ETMC) gauge field ensemble with a volume of $L^{3} \times T=48^{3} \times 96$ [7]. It features $N_{f}=2$ (i.e. mass degenerate up and down quarks) dynamical fermions at maximal twist, 


\begin{tabular}{ccccc}
\hline$\Delta \Sigma$ & up & down & strange & combined \\
\hline connected & $0.904(23)$ & $-0.311(12)$ & - & $0.594(24)$ \\
\hline disconnected & $-0.076(16)$ & $-0.076(16)$ & $-0.042(10)$ & $-0.194(25)$ \\
\hline combined & $0.828(32)$ & $-0.387(20)$ & $-0.042(10)$ & $0.400(35)$ \\
\hline \hline$A_{20}(0)=\langle x\rangle$ & up & down & strange & combined \\
\hline connected & $0.346(9)$ & $0.152(6)$ & - & $0.497(12)$ \\
\hline disconnected & $0.112(70)$ & $0.112(70)$ & $0.092(41)$ & $0.315(106)$ \\
\hline combined & $0.458(70)$ & $0.264(70)$ & $0.092(41)$ & $0.812(107)$ \\
\hline \hline$B_{20}(0)$ & up & down & strange & combined \\
\hline connected & $0.133(40)$ & $-0.149(40)$ & - & $-0.016(56)$ \\
\hline
\end{tabular}

Table 1: Renormalized results for various quark form factors including up, down and strange quarks for connected and disconnected contractions.

\begin{tabular}{cccc}
\hline quantity & \# conf. & \# source pos. & source-sink sep. \\
\hline$\Delta \Sigma$ conn. & 542 & 88 & $14 a$ \\
\hline$\Delta \Sigma$ disc. & 2137 & 100 & $14 a$ \\
\hline$A_{20}$ conn. & 580 & 16 & $16 a$ \\
\hline$A_{20}$ disc. light & 1219 & 100 & $14 a$ \\
\hline$A_{20}$ disc. strange & 2153 & 100 & $14 a$ \\
\hline$B_{20}$ & 425 & 16 & $12 a$ \\
\hline
\end{tabular}

Table 2: Statistics and source-sink seperation for the computed quark form factors.

which ensures an improved continuum limit for all here considered quantities [8]. The twisted mass parameter is set to $\mu=0.0009$ which corresponds to a physical pion mass of $m_{\pi} \approx 133 \mathrm{MeV}$. A coupling of $\beta=2.1$ is used which amounts to a lattice spacing of $a \approx 0.093 \mathrm{fm}$. The number of measurements and the source-sink separation for the different form factors can be found in Tab. 2 . If the chosen values for the source-sink separation are really sufficient to suppress possible excited state effects has to be shown in the future.

\section{Results for quark form factors}

The collected results for all available quark form factors can be found in Table 1 . This includes connected and disconnected results for up, down and strange quark form factors. A more elaborate discussion of the computation of the connected part of the form factors from the physical point ensemble can be found in Ref. [6], while the disconnected parts are presented in Ref. [9]. The charm and heavier quarks are neglected in this analysis. For the $B_{20}^{q}$ form factor there are up to this moment no disconnected results available. All necessary renormalization factors were obtained from a non-perturbative lattice calculation, $c f$. [6] and references therein. From these values we can extract the following values for the proton spin decomposition. For the quark spin we find

$$
\frac{1}{2} \Delta \Sigma=0.200(17) \quad \text { with } \quad \frac{1}{2} \Delta u=0.414(16), \quad \frac{1}{2} \Delta d=-0.194(10), \quad \frac{1}{2} \Delta s=-0.021(5) .
$$


$\frac{1}{2} \Delta \Sigma$ is slightly above the boundaries that are given from the phenomenological analysis of experimental data $0.13<\frac{1}{2} \Delta \Sigma<0.18$ [2]. For the total angular momentum of all quarks we get the following value, where we have neglected a possible disconnected contribution for the $B_{20}^{q}$ form factor

$$
J_{q}=0.398(60) \quad \text { with } \quad J_{u}=0.296(40), \quad J_{d}=0.058(40), \quad J_{s}=0.046(20) .
$$

As a notable fact the down quark barely contributes to the total angular momentum, which is in a rather good agreement with the analysis of GPDs [5], as are the results for the other quark flavors. From these results one can infer the values for the orbital angular momentum from $L_{q}=J_{q}-\frac{1}{2} \Delta \Sigma$

$$
L_{q}=0.198(62) \quad \text { with } \quad L_{u}=-0.118(43), \quad L_{d}=0.252(41), \quad L_{s}=0.067(21) .
$$

This shows that the orbital angular momentum of all quarks is of the order of the quark spin.

\section{Results for the gluon content}

On the basis of Eq. (3.4) we can try to compute the $A_{20}^{g}$ form factor on the lattice. On the lattice the gluon operator can be expressed in terms of plaquette terms, using $U_{\mu v}=\exp \left(i g a^{2} G_{\mu v}+\right.$ $O\left(a^{3}\right)$ ). We choose a representation where the gluon form factor can be extracted without applying a momentum boost to the proton.

$$
\mathscr{O}_{V}^{g}=\frac{2}{9} \frac{\beta}{a^{4}}\left(\sum_{i} \operatorname{Re}\left(U_{i 4}\right)-\sum_{i<j} \operatorname{Re}\left(U_{i j}\right)\right) .
$$

From the correlation of this operator with the nucleon two-point function, as depicted on the right side of Fig. 1, we were able to extract the gluon form factor. Up to 20 steps of 4D stout smearing [10] with $\omega=0.1315$ were used in order to remove the fluctuations due to the gauge field and thus obtain a statistically significant signal. We extract a signal from several values for the source-sink separation and obtained a bare lattice result from a combined plateau fit to these values

$$
A_{20, \text { bare }}^{g}=\langle x\rangle_{\text {bare }}^{g}=0.318(24) \quad \stackrel{\text { renormalization }}{\longrightarrow} \quad A_{20}^{g}=0.321(25) .
$$

Since the gluon operator is a singlet operator it mixes with other singlet operators, most importantly the quark singlet operator $A_{20, \text { bare }}^{q}$. The thus necessary renormalization and mixing coefficients were computed in a one-loop perturbative lattice calculation that also takes stout smearing into account and will be presented in an upcoming paper. For the $B_{20}^{g}$ form factor we are not yet able to present any results.

\section{Conclusion and Outlook}

Taking all current results into account, we present a first preliminary lattice analysis for the proton spin decomposition. This certainly is not a final lattice result since systematic effects, e.g. cut-off effects, have not thoroughly been studied and it was not possible to obtain results for all the necessary form factors. A first test of our results is the reconstruction of the total proton spin from 

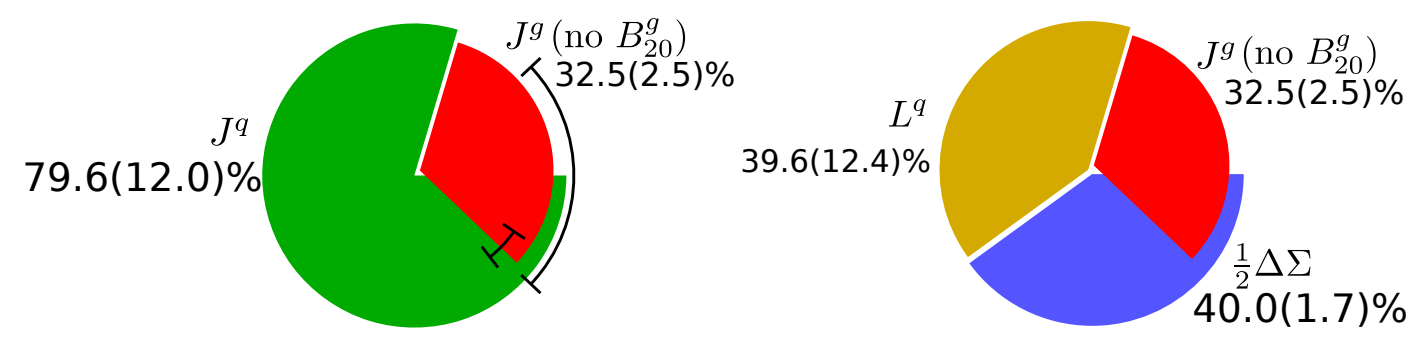

Figure 2: Graphical representation of the proton spin contribution of quarks and gluons from lattice QCD results.

lattice data from the individual form factors as shown in Fig. 2. At the moment we obtain about 110 percent of the total nucleon spin, however, with a large uncertainty of $J_{q}$, as indicated by the errorbars in the chart. If this is really a statistical effect or caused by systematic uncertainties or missing parts of the form factors is something to be explored in further studies. Nevertheless, the here presented results already provide a good qualitative study of the proton spin decomposition and certainly show that it is feasible to explore this important topic from a lattice point of view. Especially the fact that all results are extracted from a gauge ensemble with a physical pion mass will allow us to focus on further reducing other systematic effects and get closer to a good prediction for the proton spin. If this approach proves to be successful it could be easily generalized to other bound states and might in the future enable us to explore the spin structure of many hadrons.

\section{References}

[1] European MuOn collaboration, J. Ashman et al., An Investigation of the Spin Structure of the Proton in Deep Inelastic Scattering of Polarized Muons on Polarized Protons, Nucl. Phys. B328 (1989) 1.

[2] COMPASS collaboration, C. Adolph et al., The spin structure function $g_{1}^{\mathrm{p}}$ of the proton and a test of the Bjorken sum rule, Phys. Lett. B753 (2016) 18-28, [1503.08935].

[3] X.-D. Ji, Gauge-Invariant Decomposition of Nucleon Spin, Phys. Rev. Lett. 78 (1997) 610-613, [hep-ph/9603249].

[4] X.-D. Ji, Off forward parton distributions, J. Phys. G24 (1998) 1181-1205, [hep-ph/9807358].

[5] P. Kroll, Status of DVMP, DVCS and GPDs, EPJ Web Conf. 85 (2015) 01005, [1 410.4450 ].

[6] A. Abdel-Rehim et al., Nucleon and pion structure with lattice QCD simulations at physical value of the pion mass, Phys. Rev. D92 (2015) 114513, [1507.04936].

[7] ETM collaboration, A. Abdel-Rehim et al., Simulating QCD at the Physical Point with $N_{f}=2$ Wilson Twisted Mass Fermions at Maximal Twist, 1507.05068.

[8] R. Frezzotti and G. C. Rossi, Chirally improving Wilson fermions. 1. O(a) improvement, JHEP 08 (2004) 007, [hep-lat/0306014].

[9] A. Abdel-Rehim et al., Disconnected quark loop contributions to nucleon observables using $N_{f}=2$ twisted clover fermions at the physical value of the light quark mass, PoS LATTICE2015 (2015) 136, [1511.00433].

[10] C. Morningstar and M. J. Peardon, Analytic smearing of SU(3) link variables in lattice QCD, Phys. Rev. D69 (2004) 054501, [hep-lat/0311018]. 